\title{
Hospital readmissions after cardiac surgery: Is it a game worth playing?
}

\author{
Hersh Maniar, MD
}

See related article on pages $850-7$.

In their study in this issue of the Journal, "Uniform Standards Do Not Apply to Readmission Following Coronary Artery Bypass Surgery: A Multi-Institutional Study," Lancey and colleagues ${ }^{1}$ have highlighted several issues that limit the utility of the 30-day readmission rate as a suitable measure of quality when used as a pay for performance metric and call into question the practicality of its role in subsequent hospital reimbursement.

Hospital readmissions are an obvious target for Medicare because they are costly to the health care system, accounting for more than $\$ 12$ billion dollars per year, and pose significant hardship and inconvenience to patients. As a metric for scrutiny, the 30-day readmission rate is easily obtained, granular, and easy to measure. ${ }^{2}$ It is, however, not an ideal metric in isolation. A higher occurrence of readmission after an index admission is not necessarily representative of suboptimal care. In heart failure, for example, readmissions have been associated with lower 30-day mortality. A readmission penalty in this scenario would, in fact, penalize a hospital with lower mortality but whose subsequent greater number of discharged patients would be eligible for readmission. ${ }^{3}$ Moreover, the Medicare Payment Advisory Commission has stated that only $13 \%$ of readmissions are likely preventable, suggesting that even among specialties with readmission rates as high as $15 \%$ to $20 \%$, only a small percentage of patients will benefit from strategies specifically addressing readmission.

Similar to other readmission studies of cardiac surgical patients, the patients in this series were readmitted most commonly as a result of infection, arrhythmia, or an exacerbation of congestive heart failure. The date of readmission was typically within the first 2 weeks after discharge. Lancey and colleagues have additionally illustrated the difficulties that exist in identifying which specific comorbidities predispose toward readmission, and they have alluded to the

From the Department of Surgery, Washington University in Saint Louis School of Medicine, Saint Louis, Mo.

Disclosures: Author has nothing to disclose with regard to commercial support.

Received for publication Nov 24, 2014; accepted for publication Nov 25, 2014; available ahead of print Dec 24, 2014

Address for reprints: Hersh Maniar, MD, Department of Surgery, Washington University in Saint Louis School of Medicine, Campus Box 8234, 660 S Euclid Ave, St

Louis, Mo 63103 (E-mail: maniarh@wudosis.wustl.edu).

J Thorac Cardiovasc Surg 2015;149:858

$0022-5223 / \$ 36.00$

Copyright (C) 2015 by The American Association for Thoracic Surgery

http://dx.doi.org/10.1016/j.jtcvs.2014.11.069

importance of socioeconomic issues that must be considered in any future investigation of readmission. Patients with lesser financial means and health insurance are not medically savvy, are less compliant with medications, and, not surprisingly, are more likely to return to the hospital. Unfortunately, it is the hospitals that care for the economically disadvantaged that are usually less able to direct resources to prevent readmission and thus in turn are at greater risk and probably less able to tolerate future Medicare penalties. ${ }^{2,4,5}$

Although the appropriateness of the 30-day readmission metric remains controversial, it stands to remain a significant data point within pay for performance strategies, which can affect $1 \%$ to $2 \%$ of total hospital Medicare reimbursement. If the 30-day readmission rate and specifically its reduction are to be feasible and practical, targeting specific patient subgroups predisposed toward readmission and incorporating strategies that involve intervention within the first 2 weeks after discharge would seem to be paramount. ${ }^{2}$ As demonstrated in this study, readmission rates for even a single procedure such as CABG vary greatly between hospitals, and any proposed strategic interventions must be developed in the context of local hospital culture. It is, moreover, generally agreed that although reducing the readmission rate is an immediate priority to avoid financial penalty, the strategies that incorporate continuity of care, care transitions, access to primary care, and ultimately improved population health itself will be most effective in the long term. A transition from penalties to an incentive model for the latter is being considered and would likely achieve a reduction in readmissions and do so in a manner that provides lasting patient benefit and more physician buy-in. ${ }^{6}$

\section{References}

1. Lancey R, Kurlansky P, Argenziano M, Coady M, Dunton R, Greelish J, et al Uniform standards do not apply to readmission following coronary artery bypass surgery: A multi-institutional study. J Thorac Cardiovasc Surg. 2015; 149:850-7.

2. Maniar H, Bell J, Moon MR, Meyers BF, Marsala J, Lawton JS, et al. Prospective evaluation of patients readmitted after cardiac surgery: analysis of out comes and identification of risk factors. J Thorac Cardiovasc Surg. 2014 147:1013-8.

3. Gorodeski EZ, Starling RC, Blackstone EH. Are all readmissions bad readmissions? N Engl J Med. 2010;363:297-8.

4. Joynt KE, Jha AK. Thirty-day readmissions-truth and consequences. $N$ Engl J Med. 2012;366:1366-9.

5. Li Z, Armstrong EJ, Parker JP, Danielsen B, Romano PS. Hospital variation in readmission after coronary artery bypass surgery in California. Circ Cardiovasc Qual Outcomes. 2012;5:729-37. Erratum in: Circ Cardiovasc Qual Outcomes. 2012;5:e104.

6. Brown JR, Sox HC, Goodman DC. Financial incentives to improve qual ity: skating to the puck or avoiding the penalty box? JAMA. 2014;311 1009-10. 